\title{
Cribado oportunista, estrategia de prevención primaria y secundaria contra el cáncer de próstata en Lima-Perú
}

\author{
Opportunistic screening, strategy for primary and secondary prevention of prostate cancer in Lima, Peru
}

\section{Sr. Editor:}

Pow-Sang M, y Huamán M. (1), mencionan: "La detección temprana del cáncer de próstata en el Perú es muy baja, ya que los pacientes, por lo general, llegan con la enfermedad localmente avanzada o avanzada. No existen campañas de tamizaje del cáncer de próstata que nos permitan detectar esta enfermedad en estadios tempranos. Las tasas de incidencia, según el Registro de Cáncer de Lima Metropolitana, van en aumento, a pesar de existir probablemente un subregistro en nuestro país, al no contar con un registro a nivel nacional que muestre la real magnitud de esta enfermedad. Es imperativo generar programas de prevención para el diagnóstico temprano del cáncer de próstata mediante el examen digito-rectal de la próstata y el dosaje del antígeno prostático específico (PSA) en sangre".

Los datos del Instituto Nacional de Enfermedades Neoplásicas (INEN) muestran que "entre el 2006 y el 2010, el $21 \%$ fueron estadio I-II; $42 \%$ estadio III, y $37 \%$ avanzados (metastásicos)" (1). Además, "se ha estimado que el costo promedio del tratamiento de los pacientes tratados en el INEN, con cáncer de próstata localizado sometidos a prostatectomía radical con fines curativos es de S/. 5357 ; sin embargo, cuando el cáncer está localmente avanzado, el costo promedio de tratamiento por año es de S/. 10300 , y si es enfermedad avanzada es entre S/. 31000 y S/. 34 000. Estos costos aumentan considerablemente si el paciente tiene que recibir quimioterapia o tratamiento del dolor en una enfermedad avanzada" (1).

El Registro de Cáncer Poblacional de Lima Metropolitana y el Callao (2), publicó para los años 2004-2005 una observación de 2744 casos nuevos de cáncer de próstata.

En consecuencia, la asociación costo promedio del tratamiento por estadio clínico representaría un indicador de valoración para un cribado oportunista, teniendo en cuenta que: "la mayoría, si no todas las principales sociedades urológicas, han llegado a la conclusión de que, en la actualidad, el cribado de grandes poblaciones para el cáncer de próstata no es conveniente. En su lugar, debería ofrecerse una detección precoz (cribado oportunista) a pacientes bien informados" (3). Considerando estos costos fiscales por cada estadio clínico, el estado y las familias habrían gastado en tratamiento de cáncer de próstata para el periodo 2004-2005, solo en Lima Metropolitana y el Callao algo más o menos de 48 millones de soles, de los cuales 33 millones de soles serían para casos metastásicos, aquellos con muy poca oportunidad de sobrevivir más allá de cuatro a cinco años (Tabla 1, sin cribado).

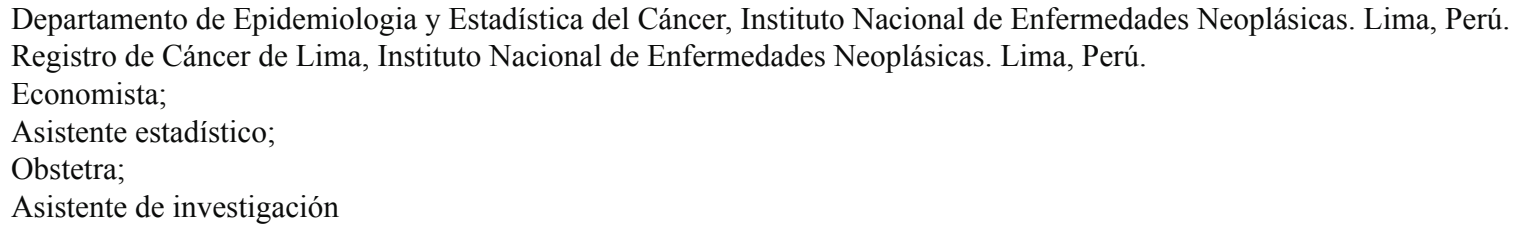


El cribado oportunista logra estructuras porcentuales mayores en estadios tempranos I y II. De esta forma se podría cambiar el perfil epidemiológico del cáncer de próstata en Lima Metropolitana y el Callao. En Estados Unidos, se calcula que el $91 \%$ de los casos nuevos de cáncer de próstata fueron diagnosticados en estadios locales o regionales, en los cuales la sobrevida relativa a 5 años se aproximó al 100\% (4). El estudio chileno de Besa P. (5), arrojó una significativa distribución porcentual de estadios tempranos los cuales deberíamos alcanzar si queremos lograr un cambio importante en el perfil epidemiológico. Esta información nos sirvió para crear nuestro segundo momento con cribado (Tabla 1).

Tabla 1. Experiencia con y sin cribado, afectados por los estadios clínicos.

\begin{tabular}{|c|c|c|c|c|}
\hline \multicolumn{5}{|c|}{ SIN CRIBADO } \\
\hline $\begin{array}{l}\text { Estadio } \\
\text { Clínico }\end{array}$ & $\begin{array}{l}\text { \% EC Estudio } \\
\text { M.Pow-Sang (1) }\end{array}$ & $\begin{array}{c}\text { Número de Casos afectado } \\
\text { por EC. Estudio RCLMC } \\
2004-2005 \text { (2) }\end{array}$ & $\begin{array}{l}\text { Costo por EC Estudio } \\
\text { M.Pow-Sang (1) }\end{array}$ & $\begin{array}{c}\text { Costos Totales } \\
\text { por EC sin } \\
\text { cribado }\end{array}$ \\
\hline $\mathrm{I}, \mathrm{II}$ & 21 & 576 & 5357 & 3085632 \\
\hline III & 42 & 1153 & 10300 & 11875900 \\
\hline IV & 37 & 1015 & 32500 & 32987500 \\
\hline & 100 & 2744 & & 47949032 \\
\hline \multicolumn{5}{|c|}{ CON CRIBADO } \\
\hline $\begin{array}{l}\text { Estadio } \\
\text { Clínico }\end{array}$ & $\begin{array}{c}\% \text { EC Estudio } \\
\text { Revista Chilena Besa } \\
\text { DC }(5)\end{array}$ & $\begin{array}{c}\text { Número de Casos afectado } \\
\text { por EC. Estudio RCLMC } \\
2004-2005 \text { (2) }\end{array}$ & $\begin{array}{l}\text { Costo por EC Estudio } \\
\text { M.Pow-Sang (1) }\end{array}$ & $\begin{array}{l}\text { Costos Totales } \\
\text { por EC con } \\
\text { cribado }\end{array}$ \\
\hline I, II & 77,63 & 2130 & 5357 & 11411306 \\
\hline III & 21,71 & 596 & 10300 & 6135941 \\
\hline \multirow[t]{2}{*}{ IV } & 0,66 & 18 & 32500 & 588588 \\
\hline & 100 & 2744 & & 18135834 \\
\hline
\end{tabular}

Por lo que sugerimos un cambio de era, un cambio del perfil epidemiológico del cáncer de próstata en Lima Metropolitana y el Callao, involucrando a instituciones comprometidas con esta problemática, mediante un cribado oportunista que pueda hallar al mayor número de casos asintomáticos, en estadios tempranos I y II, logrando curar o dar mayor y mejor chance de vida a los pacientes con este diagnóstico. Para ello es necesario lograr la participación institucional de referentes prestadores de salud oncológico que soportan una carga de población segmentada importante de pacientes con cáncer de próstata, como son: el INEN, ESSALUD, ONCOSALUD y los hospitales de las Fuerzas Armadas y de la Policía, trabajando en convenio y planificación con "PLAN ESPERANZA", quienes realizarían un cribado oportunista, como estrategia de prevención primaria y secundaria contra el cáncer de próstata en Lima-Perú, tomando un año base o año de trabajo experimental, donde las instituciones comprometidas, los primeros seis meses, realicen la prevención primaria, informando vastamente a la población de 40 años (3) a más, los factores de riesgo a contraer cáncer de próstata, sensibilizándola con la campaña de enrolamiento informado y consentido. La prevención secundaria o detección precoz, se llevará a cabo los seis meses restantes tomando en cuenta que algunos autores concluyen que "el PSA es, junto al tacto rectal (TR) y la ecografía, una herramienta útil en el diagnóstico del cáncer de próstata, siendo las tres herramientas, las que ofrecen un mejor resultado"(6).

Finalmente, si se logra una estructura porcentual de estadios clínicos como el experimentado en el estudio chileno de Besa P. (5), los costos beneficios serán importantes, tanto en lo económico como en lo social. Con la información reunida, claramente se ahorraría, cerca de 30 millones de soles y la asociación de las variables costo promedio del tratamiento por estadio clínico pondrán en valor el impacto del cribado oportunista. 


\section{Correspondencia:}

Enrique Zevallos Santillán

Correo electrónico: ezevallo@hotmail.com

\section{REFERENCIAS BIBLIOGRÁFICAS}

1. Pow-Sang M, Huamán M. Retos para el diagnóstico precoz del cáncer de próstata en el Perú. Rev perú med exp salud pública. 2013; 30(1):124-128. (Citado el 9 de diciembre del 2015) Disponible en: http://www. scielo.org.pe/scielo.php?script=sci arttext\&pid $=$ S1726-46342013000100023\&lng $=$ es $\bar{E}$ nrm $=$ iso

2. Centro de Investigación en Cáncer Maes Heller, Instituto Nacional de Enfermedades Neoplásicas. Registro de Cáncer de Lima Metropolitana 20042005. Lima, Perú: Instituto de Enfermedades Neoplásicas; Octubre 2013.

3. Heidenreich A, Bellmunt J, Bolla M, et al . Guía de la EAU sobre el cáncer de próstata: Parte I: cribado, diagnóstico y tratamiento del cáncer clínicamente localizado. Actas Urol Esp. 2011; 35(9):501-514.
4. Pow-Sang M, Destefano V, Astigueta J, et al. Cáncer de próstata en Latinoamérica. Actas Urol Esp. 2009; 33(10): 1057-1061.(Citado el 13 de diciembre 2015). Disponible en: http://scielo. isciii.es/scielo.php?script=sci_arttext\&pid=S021048062009001000005\&lng=es

5. Besa P, Rosso R, Bustos M, Borghero Y, Trucco C, Mac-Namara M. Tratamiento del cáncer de próstata con radioterapia pormodulación de intensidad, primera experiencia en Chile. Rev méd Chile. 2011; 139(11): 1451-1457. http://dx.doi.org/10.4067/S003498872011001100009

6. Acosta NM, Vera PL, Na EK, et al. Niveles séricos del antígeno prostático específico (psa) dentro de la campaña de prevención del cáncer de próstata. Mem Inst Investig Cienc Salud. 2010; 8(2): 14-19.

Recibido: 19/01/2016 